\title{
Discriminant analysis of the relationship between estimated spinal level of posterior superior iliac spine (PSIS) and spinal-pelvic parameters
}

Xi Luo

Changzheng Hospital

Yuan Wang

Changzheng Hospital

Ximing Xu

Changzheng Hospital

Kaiqiang Sun

Changzheng Hospital

Jian Zhu

Changzheng Hospital

Yanzong Lu

Changzheng Hospital

\section{Yongfei Guo}

Changzheng Hospital

Jingchuan Sun

Changzheng Hospital

Jiangang Shi ( $\sim$ brookluo@outlook.com )

Changzheng Hospital

\section{Research article}

Keywords: posterior superior iliac spine, spinal-pelvic parameters, estimated level, positioning, Fisher linear discriminant analysis

Posted Date: September 9th, 2019

DOI: https://doi.org/10.21203/rs.2.14213/v1

License: (c) (1) This work is licensed under a Creative Commons Attribution 4.0 International License.

Read Full License 


\section{Abstract}

Background: The spinal level determined by reference of posterior superior iliac spine (PSIS) will be different because of the various sagittal posture of spine-pelvis complex. The study aimed at investigating the anatomical factors affecting the estimated spinal level of PSIS from the standpoint of spine-pelvis paraments, and provided a basis for improving the accuracy of positioning.

Methods: The lumbar X-ray images of 76 patients were retrospectively analyzed. The population was classified according to the estimated level of PSIS. lumbar lordosis (LL), sacral slope (SS), pelvic incidence (PI), pelvic tilt (PT) and other parameters were measured. Then, the latent factors affecting the estimated level were filtered and obtained by One-way ANOVA and Fisher linear discriminant analysis to further summarize the imaging characteristics of different populations.

Results: Three different levels of L5 (10 cases), S1 (46 cases) and S2 (20 cases) were observed. ANOVA analysis showed that LL, SS, PT, PI, SS-PT, LL-SS and lordosis of L1-L5 (LL L1-L5) were significantly different among the three groups $(P<0.05)$. Discriminant analysis showed that $L L, S S, S S-P T$ and LL L1L5 were the main factors affecting the estimated level of PSIS $(P<0.05)$.

Conclusions: The variations of morphological parameters such as LL, SS, SS-PT and LL L1-L5 can affect the estimated level of PSIS, and the level can be predicted by the discriminant function. In the study, the

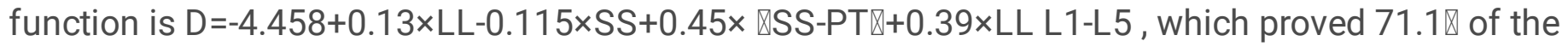
discriminant accuracy rate.

\section{Background}

Bony marker palpation is a useful method for locating spinal level in clinic. Posterior superior iliac spine (PSIS) is the most common markers for lumbosacral vertebral localization, and PSIS's lower edge is considered to correspond to the height of S2 spinous process[1]. However, in practical application, due to individual differences in anatomy, posture and palpation techniques, the actual positioning level of PSIS still fluctuates from L5 to S2, which makes it difficult to locate accurately. Therefore, by collecting lateral X-ray images of the lumbosacral region, this study attempted to apply Fisher discriminant analysis to figure out the relationship between spinal-pelvic parameters and corresponding levels of PSIS. It will provide higher accuracy for spinal manipulation if a discriminant function can be established to correct the positioning errors caused by differences of individual spinal-pelvic parameters.

\section{Methods}

\subsection{Research objects}

A retrospective analysis was performed involving 76 patients without low back pain complaints in department of orthopedics, Changzheng Hospital from January 2017 to January 2019. All patients agreed to the record of research data, and signed the informed consent. The X-ray lateral images of the 
lumbosacral region of 76 patients were collected for the subsequent measurement. Inclusion criteria: 1) clear image, no interference, including L1 vertebra and femoral head; 2) clear vertebral sequence; 3) no history of spinal surgery, no unequal length of lower limbs, no scoliosis, spondylolisthesis and traumatic lesions.

\subsection{Image measurement methods}

The spinal-pelvic parameters on X-ray films were measured by the measurement software MB Ruler. All measurements were performed by the same observer. Specific parameters are as follows:

1) The corresponding level of PSIS: a horizontal line was drew passing the most prominent point of PSIS on X-ray images, and the vertebral body passed by this line was marked as the corresponding level of PSIS.

2) Sacral slope (SS)

3) Pelvic incidence (PI)

4) Pelvic tilt (PT)

5) Lumbar lordosis (LL): the angle between the line at the upper endplate of L1 and the upper endplate of S1.

6) Lordosis of L1-L5 (LL $\left.L_{L 1-L 5}\right)$ : the angle between the line at the upper end plate of $L 1$ and the lower endplate of L5.

7) Lordosis of $L 5-S 1$ ( $\left.L L_{L 5-S 1}\right)$ : the angle between the line at the lower endplate of $L 5$ and the upper endplate of S1.

\subsection{Statistical methods}

SPSS 22.0 software was used for data analysis.

\subsubsection{Demographic Information Distribution}

One-way ANOVA and Chi-square analysis were used to analyze the age and gender differences among different groups according to the corresponding level of PSIS.

1.3.1. Filtering of spinal-pelvic parameters affecting the corresponding level of PSIS by one-way ANOVA

10 indicators including LL, SS, PI, PT, SS-PT (SS minus PT), SS/PT (SS divided by PT), LL/SS (LL divided by $S S), L_{L 1-L 5}$ and $L L_{L 5-S 1}$ were included in one-way $A N O V A$, and the indicators with statistical differences among different groups were filtered. The statistical level was $a=0.05$.

1.3.2. Fisher discriminant analysis 
Taking the PSIS positioning level as grouping variable, the indicators selected by one-way ANOVA were included in Fisher discriminant analysis. The method of "Enter independents together" was used to establish the discriminant function. The variables would be included in the final discriminant function when $P<0.05$. After obtaining the meaningful discriminant indicators, the discriminant function and its accuracy would be tested by back substitution.

\section{Results}

\subsection{General information}

In the 76 patients involved in the study, the positioning levels of PSIS were concentrated in L5, S1 and S2. Therefore, all the subjects were divided into three groups: (1) L5 group; (2) S1 group; (3) S2 group. The patients from S1, S2, and L5 group occupied $60.5 \otimes, 13.2 \bigotimes$ and $26.3 \bigotimes$ of total cases, respectively. There was no significant difference in age and gender among the three groups $(P>0.05)$ (Table 1$)$.

\subsection{One-way ANOVA of spinal-pelvic parameters}

One-way ANOVA showed that there were significant differences in the parameters of LL, SS, PI, PT, SS-PT, LL-SS and LL 1 -L5 among the three groups $(P<0.05)$. The four indicators of $L L, S S, P I$ and SS-PT in L5 group were significantly higher than those in other two groups $(P<0.05)$. Meanwhile, LL-SS and LL L1-L5 were significantly different among the three groups $(P<0.05)$, and the values were in descending order from L5, S1 to S2. PT in group S1 was significantly higher than that in group L5 $(P<0.05)$ (Table 2$)$.

\subsection{Fisher discriminant analysis}

Six parameters of LL, SS, PT, SS-PT, LL-SS and $L_{L} L_{1-L 5}$ with statistical differences in one-way ANOVA were included in the discriminant analysis. Because PI has significant correlation with PT, SS and LL[2], and is the constant characteristic value of adult pelvis[3], which indicates that PI remains unchanged with the change of posture and posture. Therefore, this study did not include PI in discriminant analysis (Table 3). The discriminant function is shown in Formula 1.

$\mathrm{D}=-4.458+0.13 \times \mathrm{LL}-0.115 \times \mathrm{SS}+0.45 \times \mathrm{SS}-\mathrm{PT}+0.39 \times \mathrm{LLL1} 1-\mathrm{L} 5 \cdots$ Formula 1

L5 group centroids $=2.424$

$\mathrm{S} 1$ group centroids $=-0.163$

$\mathrm{S} 2$ group centroids $=-0.836$

The discriminant function is applied as follows. The $D$ value of the discriminant function would be calculated, as soon as the four indicators of LL, SS, SS-PT and LL $L_{L 1-L 5}$ of a patient could be brought into the discriminant function of Formula 1. Then, the differences between the D value and the centroid of L5, S1 and S2 groups were made by subtraction, respectively. Finally, the patients were classified into the 
group which could produce the smallest difference. The correct judgment rates of L5, S1 and S2 groups were 90囚, 67.4\ and 70冈 respectively, indicating that the function held better sensitivity in discrimination of L5, but the discriminant stability between $\mathrm{S} 1$ and $\mathrm{S} 2$ groups still needed to be improved.

\section{Discussion}

\subsection{Problems in PSIS positioning}

As a means of physical examination without any tools, palpating PSIS is a quick and simple method in clinical work. McGaugh et al. utilized computed tomography (CT) three-dimensional image to confirm that $81 \rrbracket$ of the patients' lower edge of PSIS corresponds to the S2 spinous process[4]. However, PSIS also has its own application limitations, and some literatures showed that the difference of spinal-pelvic parameters can lead to the positioning error of PSIS[5-7]. Vilalle et al. held the opinions that the reason for the inconsistency of the corresponding levels of PSIS can be attributed to the difference of the pelvic shape and SS[8]. In clinical practice, we also found that the spinal-pelvic parameters would change when the patient's position changes, which indeed affected the positioning results of PSIS palpation[9, 10].

\subsection{Analysis of the PSIS positioning errors due to spinal-pelvic parameters}

In adulthood, with the pelvic shape fixed, the spatial relationship between the iliac bone and the sacrum will not change[11]. Therefore, in the same adult, the spatial relationship between the PSIS and the sacrum is constant $[12,13]$. From this point of view, it seems that if examiners utilize the PSIS located on the pelvis as bony marker to position the sacral level also located on the pelvis, the result should be fairly stable. However, in our long-term clinical work, we found that in some patients whose physiological lumbar curvature disappeared, when their LL was restored after surgery, the corresponding vertebral levels of the PSIS determined by palpation would change. This is because when positioning spinal level by PSIS, the essence of the position process is to project both posterior superior iliac spine and lumbosacral vertebrae vertically to the plane where the patient's coronal plane is located at the same time, and then determine the corresponding level according to the coincidence relationship after projection. Therefore, when the spinal-pelvic parameters change, even if the pelvic shape is fixed, the estimated level will change. As shown in Figure 1, the pelvis of $a, b$ and $c$ are identical in shape, but the locating levels are completely different. Therefore, we try to propose a hypothesis that the vertebral levels determined by palpating PSIS will drift upward or downward depending on the individual spinal-pelvic parameters, and the palpation levels of PSIS can be predicted by the spinal-pelvic parameters. The morphological and parametric characteristics of three groups of population are discussed below.

(1) PSIS positioning S1

According to Roussouly's statistics on pelvic parameters of 160 asymptomatic adults, the mean $\mathrm{PI}$ is $51.91^{\circ} \otimes 33.75^{\circ} \sim 83.72^{\circ} \otimes$, the mean PT is $11.99^{\circ} \otimes-5.06^{\circ} \sim 30.59^{\circ} \mathrm{\nabla}$, and the mean SS is $39.92^{\circ} \otimes 21.22^{\circ} \sim 65.90^{\circ} \mathrm{\otimes}[14]$. The overall profile of the pelvic parameters varies greatly among individuals, and how to determine whether the individual pelvic parameters are normal has not reached an 
agreement[15]. In this study, the average PI, PT and SS of S1 group were $47.3^{\circ}, 16.3^{\circ}$ and $30.5^{\circ}$, respectively. Compared with the other two groups, S1 group had the highest coincidence with the asymptomatic adults in Roussouly' s statistics. The PI, PT and SS of S1 group all located within the range of Roussouly's statistical results, and no extreme values appeared. This result showed that for the population of PSIS positioning S1, who accounted for the highest ratio (60.5区) of the total number of the three groups' patients, their pelvic parameters were similar to those of asymptomatic people. To summarize, S1 group could be considered as the standard profile of healthy people (Figure 2).

(2) PSIS positioning L5

One-way ANOVA showed that the pelvic parameters of L5 group were significantly different from those of asymptomatic people, and the values of LL, SS, PI and SS-PT were significantly increased $(P<0.05)$. Based on the images, we summarize two prominent characteristics of the patients in L5 group: 1) the pelvis is upright, the PSIS at the posterior part of pelvis is raised to a higher position, which make it easy to approach the higher spine level; 2) the lumbar lordosis and sacrum tilt are relative obvious, making the lumbar and sacral vertebrae form a "big S" shape centering on the L5/S1 interface. Because the L5 and $\mathrm{S} 1$ are at the junction of two arcs with opposite bending directions, the horizontal distance between them is larger and the vertical distance is smaller, which further increases the chance of PSIS approaching L5 vertebral bodies (Figure 3).

From the standpoint of parameters to analyze the image characteristics of $L 5$ group, on the one hand, the PI value of L5 group is higher, which indicates that the pelvic anatomy of L5 group is different from that of the majority. The angle between sacral long axis and pelvic long axis is larger, and the sacral tilt is obvious, which leads to higher SS value. Because the spine is the body's load-bearing axis structure, the huge gravity load on the lumbar spine will be transferred to the sacrum at the L5/S1 interface, so there will be a force along the $\mathrm{S} 1$ upper endplate obliquely forward and downward. If this component increases, the lumbar spine will have to increase lordosis for compensate to reduce the component in order to reduce the risk of spondylolisthesis. Therefore, because of the high PI value of $L 5$ group, the consequent higher SS value and the greater slip force along the oblique direction of L5/S1 interface finally result in the compensatory increase of LL. On the other hand, because of the erect pelvis in L5 group, we tried to use one or more indicators to quantify pelvic posture in our study. We inferred that the pelvis has two hinges on the sagittal plane, one is the hip joint, and the other is the S5/S1 interface, which can rotate around the coronal axis. Therefore, in the pelvic parameters, neither SS nor PT can fully reflect the pelvic posture alone. Hresko used cluster analysis to divide patients into high SS/low PT group and low SS/high PT group[16]. The former had erect pelvis, while the latter had backward pelvis. Based on this, we tried to introduce a new parameter to quantify the difference between SS and PT to predict the corresponding level of PSIS. At first, we chose SS/PT (the quotient of SS and PT) and SS-PT (the algebraic difference of SS and PT) as two new parameters to study the size relationship. The results of One-way ANOVA proved that SS-PT had statistical differences among groups, and SS-PT was also included as a parameter in the final discriminant function. Therefore, we believed that SS-PT can be used 
to quantify pelvic posture and incorporated in discriminant analysis to predict the corresponding levels of PSIS.

\section{(3) PSIS positioning S2}

Except for LL-SS and $L_{\mathrm{L} 1-\mathrm{L} 5}$, the values of other spinal-pelvic parameters in $\mathrm{S} 2$ and $\mathrm{S} 1$ group presented high degree of resemblance ( $P \otimes 0.05)$. This indicated that there was no significant difference in overall lumbosacral curvature and pelvic morphology between S2 and S1 group. What made us interested was that the curvature formed by five lumbar vertebrae contributed little to the overall lumbosacral curvature in S2 group, according to image characteristics. In the images, the lumbar radian of S2 group was smaller and the overall trend was straighter (Figure 4), although their LL had no significant difference (Pख0.05).

Normal LL plays a key role in maintaining the sagittal balance of the spine. When $L L$ decreases, pelvis retroversion will come into being as compensatory changes in order to prevent trunk anteversion. At the same time, PSIS falls back together, resulting in reduction of its positioning level, making PSIS closer to S2[17]. However, in this study, there were no significant differences in LL, SS, and PT between S2 and S1 groups. We inferred that it could be attributed to the fact that the samples were from people without lumbar diseases, and the loss of lumbar lordosis and the compensation of pelvic retroversion were slight. Meanwhile, the individual difference of PI would also influence the values of PT and SS, which blurred the discrimination between S1 and S2 group.

We introduced LL-SS (the difference between LL and SS), LL/SS (the quotient between LL and SS), LL L1$L 5$ and $L L_{L 5-S 1}$ to extract lumbar curvature from the overall lumbosacral curvature, making the loss of lumbar lordosis be monitored more sensitively. Finally, LL-SS and LL $L_{L 1-L 5}$ in S2 group were both significantly lower than those in $\mathrm{S} 1$ group, but the final discriminant function only included $\mathrm{LL}_{\mathrm{L} 1-\mathrm{L} 5}$, which confirmed the effect of smaller lumbar curvature on PSIS positioning.

\section{Conclusions}

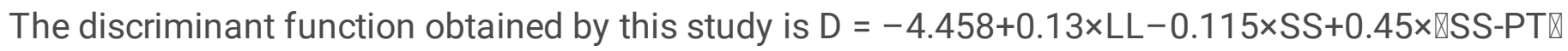
$+0.39 \times L L_{L 1-L 5}$, and the group centroids of $L 5, S 1$, and $S 2$ are $2.424,-0.163$ and -0.836 , respectively, which proved $71.1 \otimes$ of the discriminant accuracy rate. After substituting a certain patient's LL, SS, SS-PT, and $L L_{L 1-L 5}$ into the function, comparing the $D$ value to each group centroid to get the algebraic difference, and the corresponding level of PSIS can be obtained when the difference is smallest. By utilizing the discriminant function, the corresponding relationship between spinal level and spinal-pelvic parameters can be established.

\section{Abbreviations}

PSIS: Posterior superior iliac spine

SS: Sacral slope 
PI: Pelvic incidence

PT: Pelvic tilt

LL: Lumbar lordosis

$\mathrm{LL}_{\mathrm{L1} 1-\mathrm{L5}}$ : Lordosis of L1-L5

$L_{L 5-S 1}$ : Lordosis of L1-L5

CT: computed tomography

\section{Declarations}

\section{Ethics approval and consent to participate}

All procedures performed in studies involving human participants were in accordance with the ethical standards of the Ethics Committee of Changzheng Hospital.

\section{Consent for publication}

Informed consent was obtained from all individual participants included in the study.

\section{Availability of data and material}

Data sharing is not applicable to this article as no datasets were generated or analyzed during the current study.

\section{Competing interests}

The authors declare that they have no competing interests.

\section{Funding}

This study was funded by

Innovation Fund for College Students of Second Military Medical University (No. FH2017077) by Xi Luo (first author)

Innovation Fund for College Students of Second Military Medical University (No. MS2016045) by Xi Luo (first author) 
Because the study was carried out without laboratory consumables and reagent, the role of funding only supported imaging collection and data measurement.

\section{Authors' contributions}

"XL analyzed and interpreted the patient data, and was a major contributor in writing the manuscript. YW and XX performed the examination of the data, and substantively revised the manuscript. KS and JZ conducted the acquisition of data. YL have helped draft the work. YG and JC (Jingchuan Sun) proposed the ieda of sutdy design. JS (Jiangang Shi) finished the final assessment of the manuscript. All authors read and approved the final manuscript."

\section{Acknowledgements}

Not applicable.

\section{References}

1.Atlihan D, Bozkurt M, Turanli S, Dogan M, Tekdemir I, Elhan A: Anatomy of the posterior iliac crest as a reference to sacral bar insertion. Clin Orthop Relat Res 2004(418):141-145.

2.Mac-Thiong JM, Labelle H, Berthonnaud E, Betz RR, Roussouly P: Sagittal spinopelvic balance in normal children and adolescents. Eur Spine J 2007, 16(2):227-234.

3.Legaye J, Duval-Beaupère G, Hecquet J, Marty C: Pelvic incidence: a fundamental pelvic parameter for three-dimensional regulation of spinal sagittal curves. Eur Spine J 1998, 7(2):99-103.

4.McGaugh JM, Brismee JM, Dedrick GS, Jones EA, Sizer PS: Comparing the anatomical consistency of the posterior superior iliac spine to the iliac crest as reference landmarks for the lumbopelvic spine: a retrospective radiological study. Clin Anat 2007, 20(7):819-825.

5.Furness G, Reilly MP, Kuchi S: An evaluation of ultrasound imaging for identification of lumbar intervertebral level. Anaesthesia 2002, 57(3):277-280.

6.Broadbent CR, Maxwell WB, Ferrie R, Wilson DJ, Gawne-Cain M, Russell R: Ability of anaesthetists to identify a marked lumbar interspace. Anaesthesia 2000, 55(11):1122-1126.

7.Kim HW, Ko YJ, Rhee WI, Lee JS, Lim JE, Lee SJ, Im S, Lee JI: Interexaminer reliability and accuracy of posterior superior iliac spine and iliac crest palpation for spinal level estimations. J Manipulative Physiol Ther 2007, 30(5):386-389.

8.Rapha? L V, Nicolas L, Ludovic R, Alexandre T, Wafa S, Pierre G: Radiographic analysis of the sagittal alignment and balance of the spine in asymptomatic subjects. Journal of Bone \& Joint Surgery American 
Volume 2005, 87(2):260.

9.Tarantino U, Fanucci E, lundusi R, Altobelli S, Gasbarra E, Simonetti G, Manenti G: Lumbar spine MRI in upright position for diagnosing acute and chronic low back pain: statistical analysis of morphological changes. Journal of Orthopaedics \& Traumatology Official Journal of the Italian Society of Orthopaedics \& Traumatology 2013, 14(1):15-22.

10.Park SA, Kwak DS, Cho HJ, Min DU: Changes of spinopelvic parameters in different positions. Arch Orthop Trauma Surg 2017, 137(9):1223-1232.

11.Jacob HA, Kissling RO: The mobility of the sacroiliac joints in healthy volunteers between 20 and 50 years of age. Clin Biomech (Bristol, Avon) 1995, 10(7):352-361.

12. Hanson DS, Bridwell KH, Rhee JM, Lenke LG: Correlation of pelvic incidence with low- and high-grade isthmic spondylolisthesis. Spine (Phila Pa 1976) 2002, 27(18):2026-2029.

13.Jean L: Influence of age and sagittal balance of the spine on the value of the pelvic incidence. Eur Spine J 2014, 23(7):1394-1399.

14.George W, Michael H: Classification of the normal variation in the sagittal alignment of the human lumbar spine and pelvis in the standing position. Spine (Phila Pa 1976) 2005, 30(13):1558.

15.Kumar M, Baklanov A, Chopin D: Correlation between sagittal plane changes and adjacent segment degeneration following lumbar spine fusion. Eur Spine J 2001, 10(4):314-319.

16. Hresko MT, Labelle H, Roussouly P, Berthonnaud E: Classification of high-grade spondylolistheses based on pelvic version and spine balance: possible rationale for reduction. Spine (Phila Pa 1976) 2007, 32(20):2208-2213.

17.Tetsuya K, Yuji A, Takeo M, Naoki T: A longitudinal study of congruent sagittal spinal alignment in an adult cohort. Spine (Phila Pa 1976) 2004, 29(6):671-676.

\section{Tables}

Table 1 Demographics

\begin{tabular}{lcccc}
\hline & L5 & S1 & S2 & P \\
\hline No. of patients & 10 & 46 & 20 & \\
\hline Gender & & & & $\square 0.05$ \\
\hline \multicolumn{1}{c}{ Female } & 6 & 22 & 12 & \\
\hline Male & 4 & 24 & 8 & \\
\hline Age $₫$ years $₫$ & $35.9 \pm 9.4$ & $41.3 \pm 15.7$ & $40.1 \pm 13.2$ & $\square 0.05$ \\
\hline
\end{tabular}


Table 2 Spine-pelvis index One-way ANOVA

\begin{tabular}{lllllll}
\hline & \multicolumn{1}{c}{ L5 } & \multicolumn{1}{c}{ S1 } & \multicolumn{1}{c}{ S2 } & \multicolumn{1}{c}{ P1 } & P2 & P3 \\
\hline LL (degree) & $68.8 \pm 12.4$ & $45.7 \pm 11.1$ & $40.7 \pm 4.9$ & $0.000^{*}$ & 0.071 & $0.000^{*}$ \\
\hline SS (degree) & $50.1 \pm 16.7$ & $30.5 \pm 9.5$ & $30.3 \pm 7.3$ & $0.000^{*}$ & 0.960 & $0.000^{*}$ \\
\hline PI (degree) & $60.1 \pm 21.5$ & $47.3 \pm 13.0$ & $43.3 \pm 13.3$ & $0.012^{*}$ & 0.298 & $0.003^{*}$ \\
\hline PT (degree) & $9.5 \pm 6.3$ & $16.4 \pm 9.0$ & $12.7 \pm 9.2$ & $0.026^{*}$ & 0.112 & 0.354 \\
\hline SS-PT (degree) & $40.6 \pm 14.0$ & $14.1 \pm 13.3$ & $17.7 \pm 9.2$ & $0.000^{*}$ & 0.280 & $0.000^{*}$ \\
\hline LL-SS (degree) & $18.7 \pm 5.5$ & $15.2 \pm 4.9$ & $10.4 \pm 4.9$ & $0.044^{*}$ & $0.001^{*}$ & $0.000^{*}$ \\
\hline SS/PT & $9.5 \pm 8.5$ & $4.3 \pm 7.8$ & $5.9 \pm 10.1$ & 0.084 & 0.495 & 0.272 \\
\hline LL/SS & $1.5 \pm 0.4$ & $1.6 \pm 0.3$ & $1.4 \pm 0.3$ & 0.539 & 0.069 & 0.474 \\
\hline LL & $48.2 \pm 14.2$ & $31.6 \pm 9.6$ & $26.2 \pm 8.4$ & $0.000^{*}$ & $0.049^{*}$ & $0.000^{*}$ \\
\hline LL $_{\text {L5-S1 }}$ (degree) & $19.9 \pm 12.9$ & $14.8 \pm 5.4$ & $17.0 \pm 6.7$ & 0.043 & 0.239 & 0.303 \\
\hline
\end{tabular}

*P is less than 0.05. P1 means comparing L5 and S1هP2 means comparing S1 and S2『P3 means comparing L5 and $S 2 \rrbracket a=0.05$.

Eigenvalue Wilks' $\lambda \quad \mathrm{c}^{2} \quad \begin{aligned} & \text { Canonical } \\ & \text { correlation }\end{aligned} \quad \mathrm{P} \quad \begin{aligned} & \text { Average } \\ & \text { accuracy }\end{aligned}$

\begin{tabular}{lllllll}
1.013 & 0.421 & 61.800 & 0.709 & 8 & $0.000^{*}$ & $71.10 \%$ \\
\hline
\end{tabular}

\section{Figures}

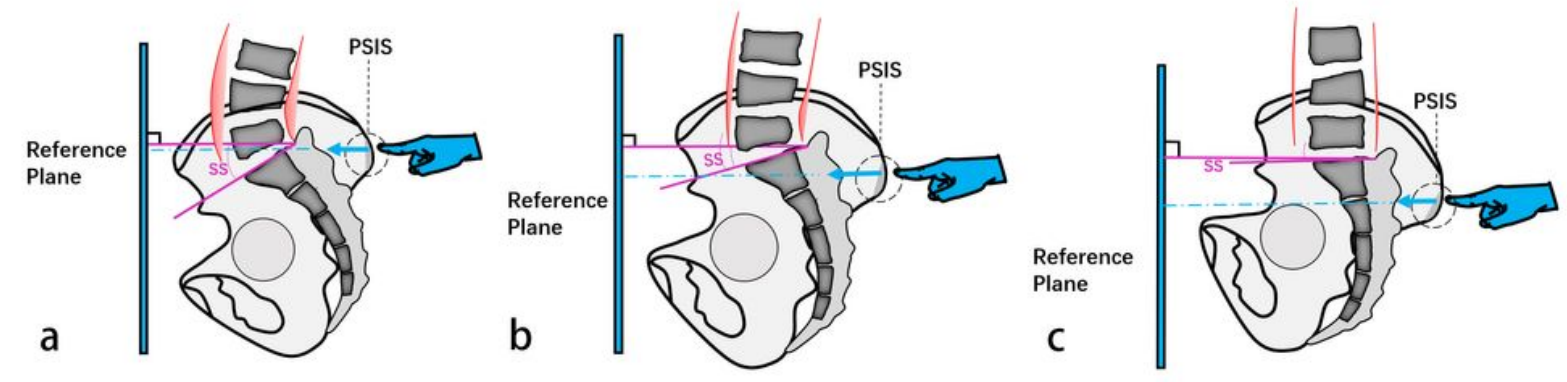

Figure 1

Three schematic diagrams with different positioning level of PSIS are completely identical in pelvic morphology, due to their different pelvic-spinal parameters. a: Palpating PSIS to position L5 level; b: Palpating PSIS to position S1 level; c: Palpating PSIS to position S2 level. 

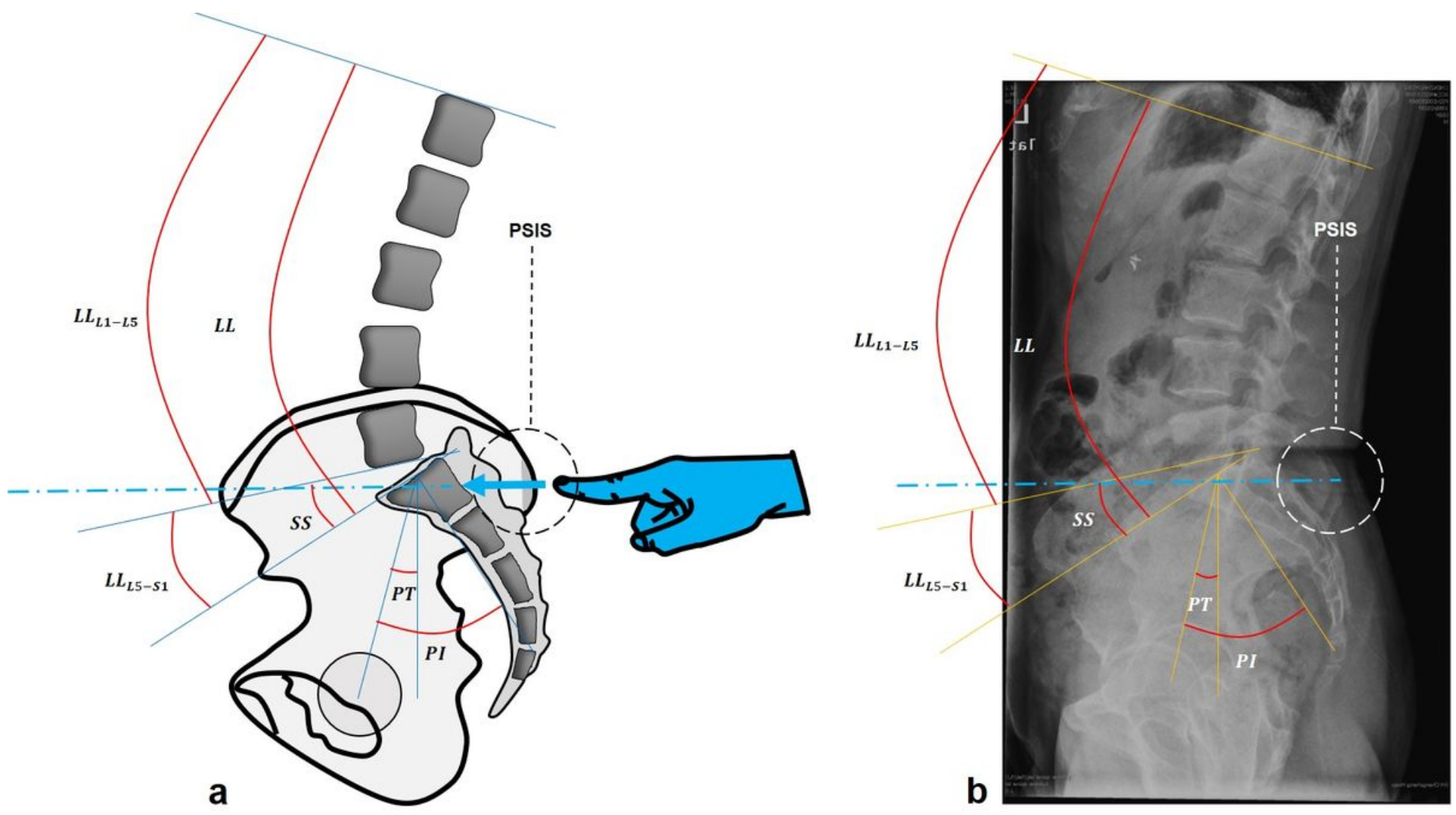

Figure 2

Palpating PSIS to position S1 level. a: schematic diagram of spine-pelvis complex without extreme numerical value of spinal-pelvic parameters. $\mathrm{LL}=56^{\circ}, \mathrm{SS}=36^{\circ}, \mathrm{PT}=11^{\circ}, \mathrm{PI}=47^{\circ}, \mathrm{LLL1}-\mathrm{L} 5=36^{\circ}, \mathrm{LLL5}-\mathrm{S} 1$ $=20^{\circ}, \mathrm{LL}-\mathrm{SS}=20^{\circ}, \mathrm{LL} / \mathrm{SS}=1.5, \mathrm{SS}-\mathrm{PT}=25^{\circ}, \mathrm{SS} / \mathrm{PT}=3.3 \mathrm{~b}$ : $\mathrm{X}$-ray images of a patient with PSIS positioning S1. 

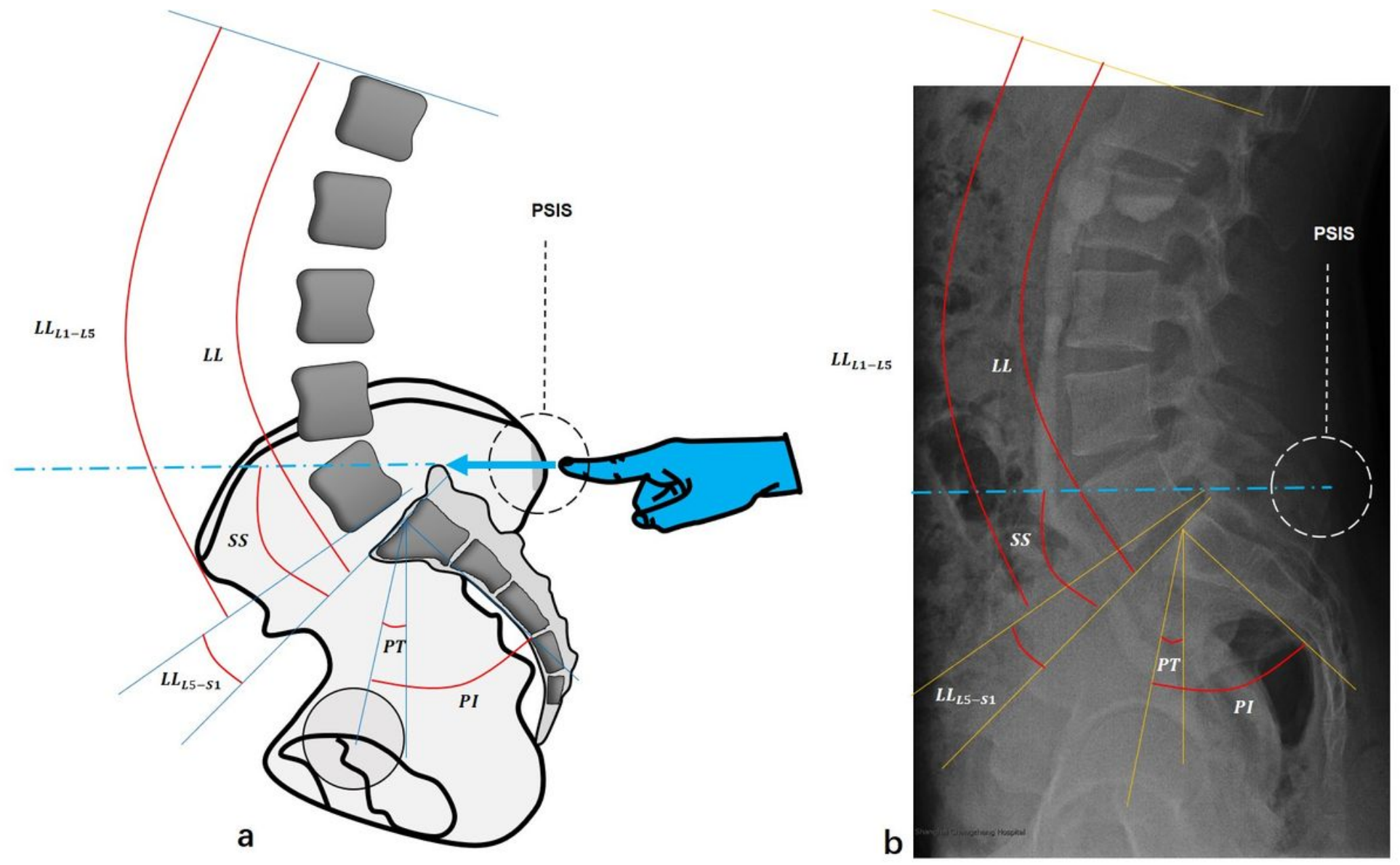

Figure 3

Palpating PSIS to position L5 level. a: schematic diagram of spine-pelvis complex characterized by higher $\mathrm{LL}$, higher SS and higher SS-PT. LL $=69^{\circ}, \mathrm{SS}=56^{\circ}, \mathrm{PT}=10^{\circ}, \mathrm{PI}=66^{\circ}, \mathrm{LLL1}-\mathrm{L} 5=55^{\circ}, \mathrm{LLL5}-\mathrm{S} 1=14^{\circ}, \mathrm{LL}-\mathrm{SS}=13^{\circ}$, $\mathrm{LL} / \mathrm{SS}=1.2, \mathrm{SS}-\mathrm{PT}=46^{\circ}, \mathrm{SS} / \mathrm{PT}=5.6$. b: X-ray images of a patient with PSIS positioning L5. 

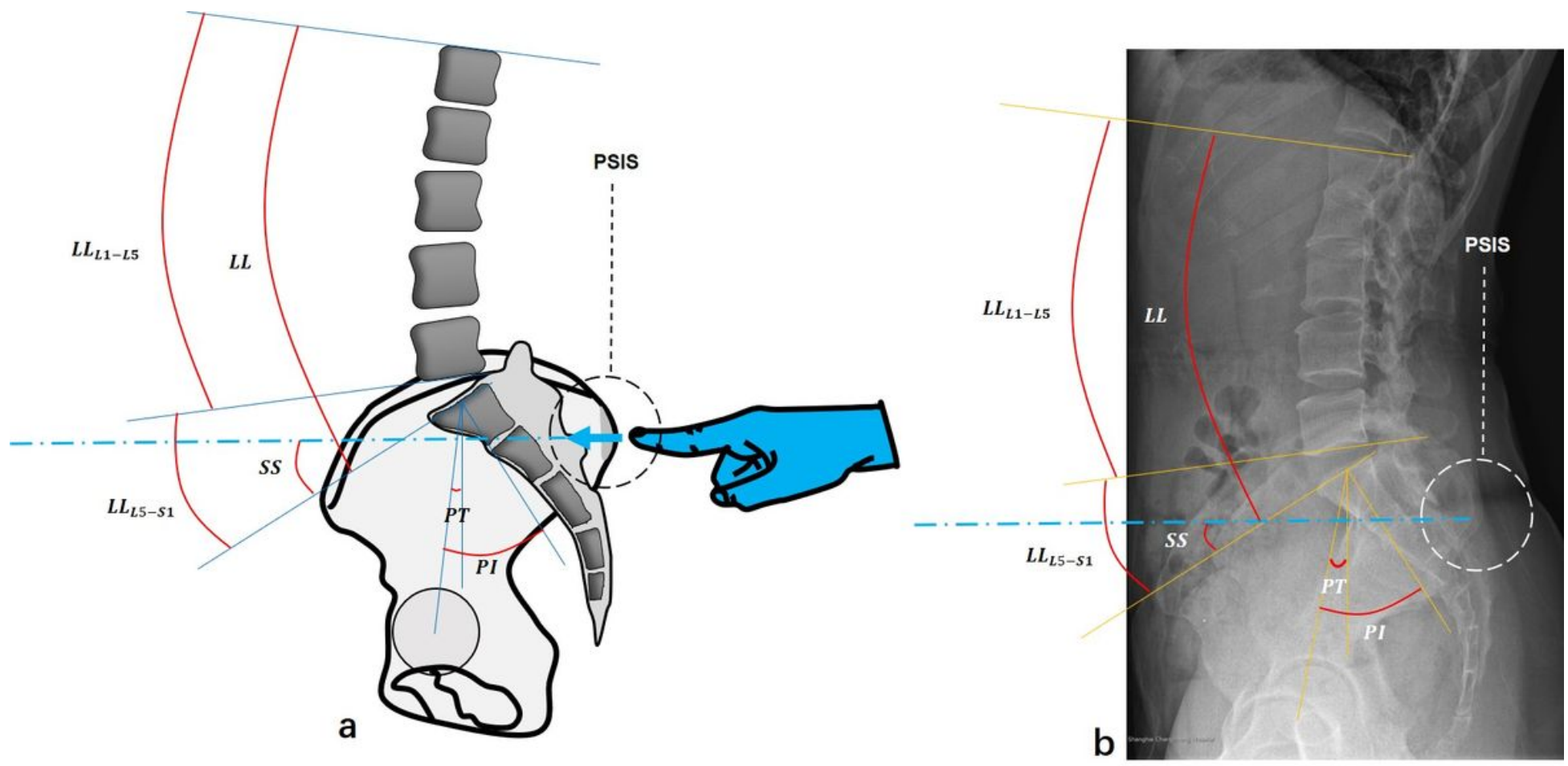

Figure 4

Palpating PSIS to position S2 level. a: schematic diagram of spine-pelvis complex characterized by lower LL-SS and lower LLL1-L5. LL $=40^{\circ}, S S=29^{\circ}, P T=13^{\circ}, P I=42^{\circ}, \mathrm{LLL1}-\mathrm{L5}=23^{\circ}, \mathrm{LLL5}-\mathrm{S} 1=17^{\circ}, \mathrm{LL}-\mathrm{SS}=11^{\circ}$, $\mathrm{LL} / \mathrm{SS}=1.4, \mathrm{SS}-\mathrm{PT}=16^{\circ}, \mathrm{SS} / \mathrm{PT}=2.2$. b: $\mathrm{X}$-ray images of a patient with PSIS positioning $\mathrm{S} 2$. 